\title{
Cross-sectional echocardiographic assessment of conditions with atrioventricular valve leaflets attached to the atrial septum at the same level
}

\author{
JEFFREY F SMALLHORN, GEORGE R SUTHERLAND^, ROBERT H ANDERSON, \\ FERGUS J MACARTNEY
}

From The Hospital for Sick Children, Great Ormond Street, London; and Freeman Hospital, Newcastle upon Tyne

SUMMARY Ninety two patients with both atrioventricular valves attached to the atrial septum, roofing a perimembranous inlet ventricular septal defect, were assessed by cross-sectional echocardiography. In the group, 42 had an isolated perimembranous inlet ventricular septal defect, 31 had atrioventricular discordance, nine an atrioventricular septal defect with intact interatrial septum, and 10 a straddling atrioventricular valve. In all but those with an atrioventricular septal defect the left atrioventricular valve had the appearance of a morphologically mitral valve. In the former lesion the atrioventricular junction was "sprung" and a cleft between the anterior and posterior bridging leaflets was identified in all. A straddling valve was identified by tensor apparatus from one atrioventricular valve in both ventricular chambers. Atrioventricular discordance was diagnosed by identifying the systemic and pulmonary venous atria and then assessing the morphology of the draining atrioventricular valves. Thus, with cross-sectional echocardiography, the constellation of abnormalities that give rise to lack of offsetting of the atrioventricular valves can be reliably identified.

One of the most characteristic cross-sectional echocardiographic features of the normal atrioventricular junction is offsetting of the mitral and tricuspid valves at their attachment to the inlet ventricular septum, with the right sided valve being displaced more towards the apex than the left. Right to left inversion of this normal arrangement has been held to be characteristic of atrioventricular discordance. ${ }^{2} \mathrm{~A}$ more common abnormality, however, is lack of any offsetting of the atrioventricular valves seen in the apical and subcostal four chamber cuts. Both atrioventricular valves are attached to the interatrial septum at the same level. This finding is of course characteristic of a double inlet atrioventricular connection, but in this case its importance is trivial compared with the other abnormalities present.

Of considerably greater interest is the situation in which each atrium connects to its own ventricular chamber (biventricular atrioventricular connection)

^Present address: Freeman Hospital, High Heaton, Newcastle upon Tyne.

RHA and FJM are supported by the British Heart Foundation and respectively, the Joseph Levy and Vandervell Foundations, and RHA is employed at the Cardiothoracic Institute, Brompton Hospital.

Accepted for publication 21 May 1982 and the two atrioventricular valves, attached to the atrial septum at the same level, roof a ventricular septal defect. This must, of necessity, involve the inlet ventricular septum. Defects of this type, which are roofed by atrioventricular valve tissue, may be termed perimembranous inlet defects. ${ }^{3}$

This study was carried out in order to determine which conditions would give rise to this appearance, how they might be distinguished from one another, and whether the atrioventricular connection could be determined in the absence of offsetting of the atrioventricular valves.

\section{Subjects and methods}

All the cases studied were drawn from the routine work at The Hospital for Sick Children, Great Ormond Street, and Freeman Hospital, Newcastle upon Tyne. The patients were studied with either an Advanced Technology Laboratory Mechanical Sector Scanner or a Toshiba phased array system. Only those cases with biventricular atrioventricular connection were included in the study. A morphologically right ventricle was determined by the presence of septal insertion of the tricuspid valve, a trileaflet appearance 
of the valve, and the presence of a moderator band. In patients with a straddling tricuspid valve the presence of chordae attached to the crest of the septum could not be used as a reliable indicator of right ventricular morphology. In these patients the other features were used to identify a morphological right ventricle. A morphologically left ventricle was determined by a smooth interventricular septum free of septal insertions, and a bileaflet pattern of the mitral valve.

Initially, an apical four chamber cut was obtained to visualise the level of the atrioventricular valves and to determine the integrity of the primum atrial septum. If a defect in the latter was present, patients were then excluded from the study; those included were the cases where the left and right atrioventricular valves roofed the entire defect. The pattern of movement of the valve leaflets was also noted, in an attempt to detect the presence of straddling. Any overriding of the atrioventricular valve annulus associated with the straddling was also noted in this cut.

The transducer beam was then angled towards the patient's right and slightly posteriorly, following the relation of the tip of the interventricular septum to the central fibrous body. This enabled identification of minor degrees of overriding associated with a straddling right atrioventricular valve, which were not visualised in a four chamber cut. The transducer was then moved back into a precordial four chamber cut. From that position the probe was angled slightly anteriorly to assess the relation of the posterior great artery to the atrioventricular junction. With clockwise rotation of the scanhead a short axis cut at the level of the posterior great artery was then obtained. The transducer was then angled posterolaterally towards the left ventricular apex. During the scan the shape of the left atrioventricular valve was noted in an attempt to identify its morphology. The presence or absence of a "cleft" in this valve was also assessed in this view. Finally, the scan head was placed so as to obtain a long axis cut and the left ventricular outflow tract was studied, paying attention to the insertion and movement of the left sided atrioventricular valve.

Next a subcostal four chamber cut was obtained. Firstly, the relation of the posterior part of the interventricular septum to the central fibrous body was noted, then the insertion of the atrioventricular valve leaflets in the appropriate chambers. In addition in the same position the drainage of the pulmonary veins could be seen and the integrity of the interatrial septum checked. With anticlockwise rotation of the scan head into a long axis cut the inferior vena cava could be followed into the systemic venous atrium.

Then, with clockwise rotation of the transducer from the four chamber position a subcostal long axis cut could be obtained. In this view the atrioventricular valves and mode of insertion could be noted. Dur- ing real-time the pattern of movement could be seen in a short axis and the morphology of the atrioventricular valves identified.

Chambers and valves are named according to their morphology, not position.

\section{Results}

Atrial situs was assessed in all patients by filter films of the bronchi ${ }^{4}$ or by observing the position of the aorta and inferior vena cava relative to the spine in a short axis subcostal abdominal cut, ${ }^{5}$ and noting the drainage of the inferior vena cava and pulmonary veins in the subcostal four chamber cut.

One case had situs inversus, two had left isomerism, with the remainder having situs solitus. In all cases the echocardiographic prediction corresponded with the filter films.

PERIMEMBRANOUS INLET VENTRICULAR SEPTAL DEFECT

Atrioventricular concordance (42 cases)

In the apical four chamber cut, in all cases the atrioventricular valves were at the same level, roofing a defect in the inlet septum (Fig. 1). The ostium primum septum was intact in every patient, being in continuity with the atrioventricular valves. During real-time the morphologically mitral valve appeared to cross the beam of the transducer inserting towards the apex of the left ventricle. The tricuspid valve, however, opened towards the centre of the ventricle, the orifice being clearly visualised. A septal insertion was seen in only seven cases. With slight anterior angulation of the transducer, the wedged appearance of the posterior great artery between the atrioventricular valves was present in all cases (Fig. 2a and 3a).

In the parasternal long axis cut, the anterior leaflet of the mitral valve inserted towards the apex of the ventricle, the left ventricular outflow tract having a normal appearance (Fig. 4a).

In the subcostal four chamber cut the draining atrioventricular valves could be identified. A morphologically mitral valve appeared to insert into the anterolateral and posteromedial papillary muscle group, the septal surface of the ventricle being free of chordal attachments. A tricuspid valve, on the other hand, had a septal insertion.

The interventricular septum had an oblique orientation reflecting the normal left sided position of the cardiac apex. The ventricular septal defect was visualised in only 13 cases in this cut.

In the subcostal long axis cut the left ventricular outflow tract appeared to be normal, the mitral valve inserting towards the apex of the left ventricle while the tricuspid attachment to the interventricular septum was present in all cases. It was only in this view 

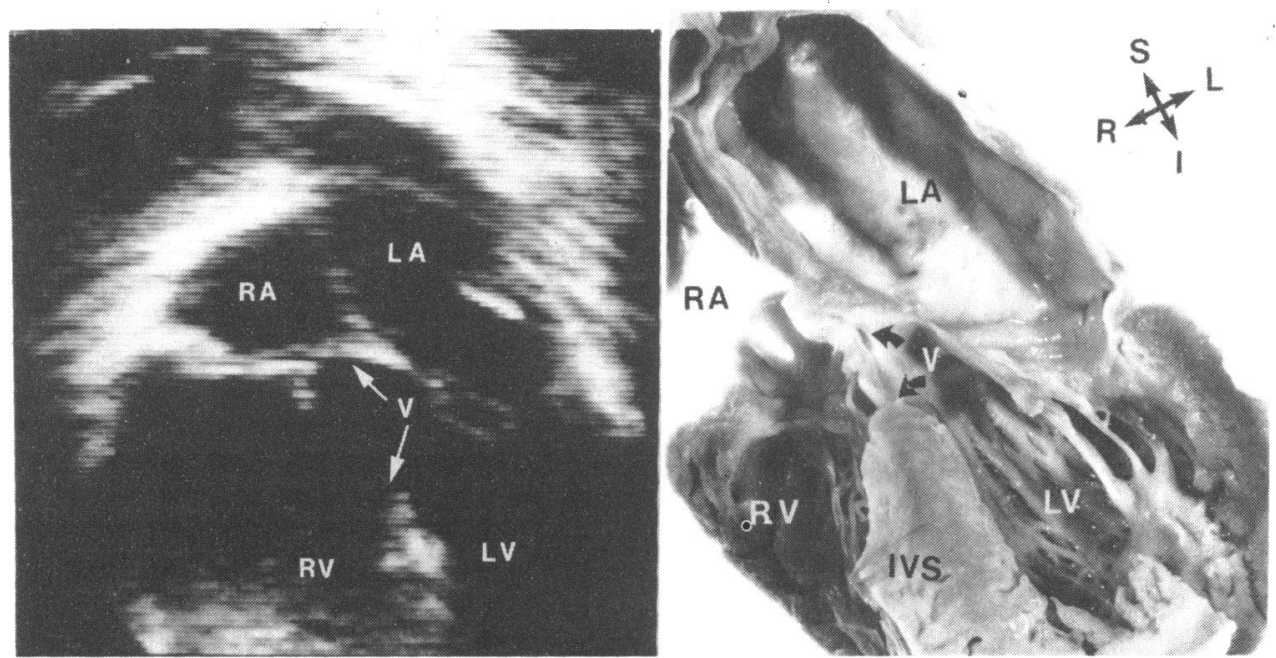

Fig. 1 Precordial four chamber cut from a case with a perimembranous inlet ventricular septal defect. The atrioventricular valves are at the same level, roofing the defect. The specimen on the right shows the echocardiographic features. IVS, interventricular septum; $L A$, left atrium; $L V$, left ventricle; $R A$, right atrium; $R V$, right ventricle; $V$, ventricular septal defect.

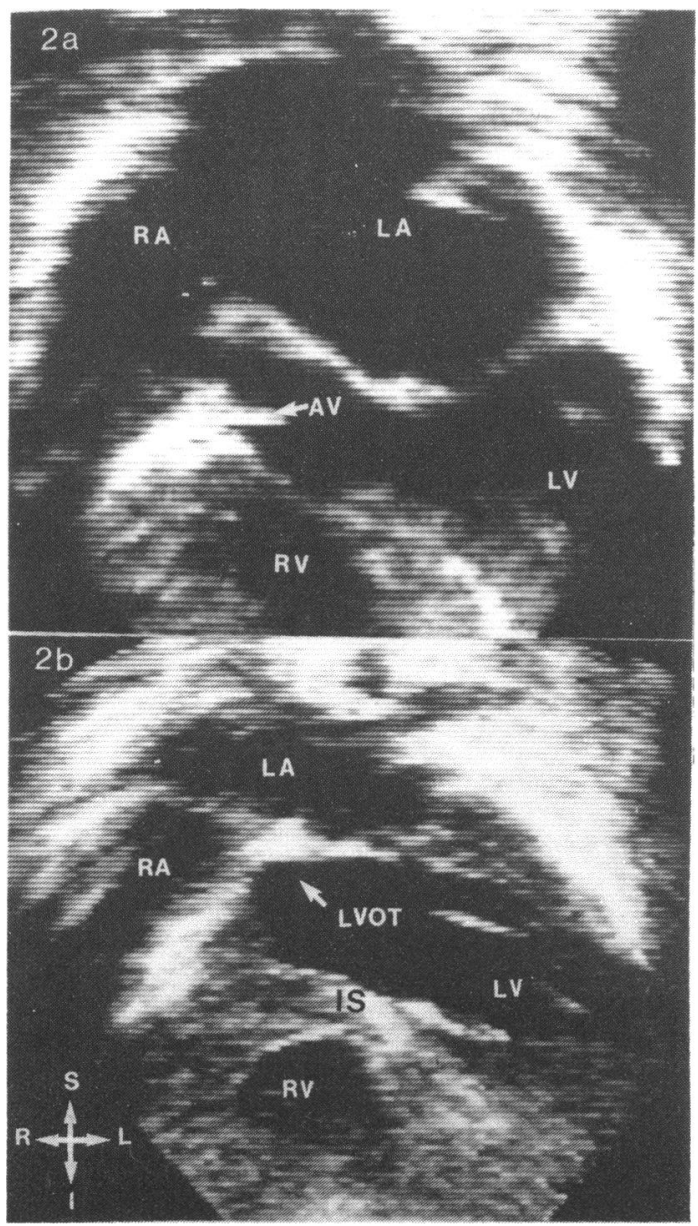

Fig. 2 (a) The upper picture is a precordial four chamber aortic root cut in a case with a perimembranous inlet ventricular septal defect and normal atrioventricular junction. The aortic valve indicated by the arrow is seen in this cut. (b) The lower picture is a similar cut in a case with an atrioventricular septal defect. Despite the cut being more anterior than the above picture, the aortic valve is not seen. $A V$, aortic valve; $I S$, infundibular septum; LVOT, left ventricular outflow tract (see Fig. I for remaining abbreviations). 

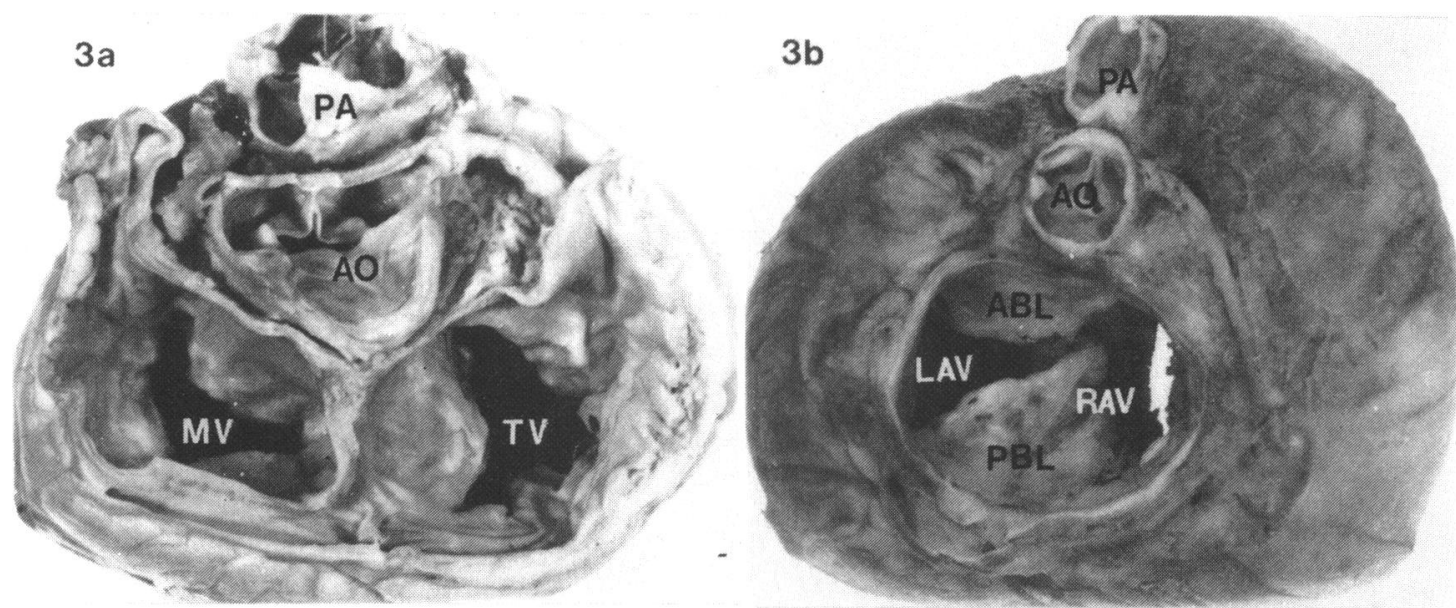

Fig. 3 (a) The specimen on the left is a normal heart viewed from above with the atria removed. There is normal morphology of the atrioventricular valves and the aorta occupying its wedged position. (b) The specimen on the right is from a case with an atrioventricular septal defect. The "cleft" between the anterior and posterior bridging leaflets points towards the right ventricle. In addition the whole atrioventricular junction is sprung with the aorta no longer occupying its wedged position. $A O$, aorta; $A B L$, anterior bridging leaflet; $M V$, mitral valve; $P A$, pulmonary artery; $P B L$, posterior bridging leaflet; $T V$, tricuspid valve.

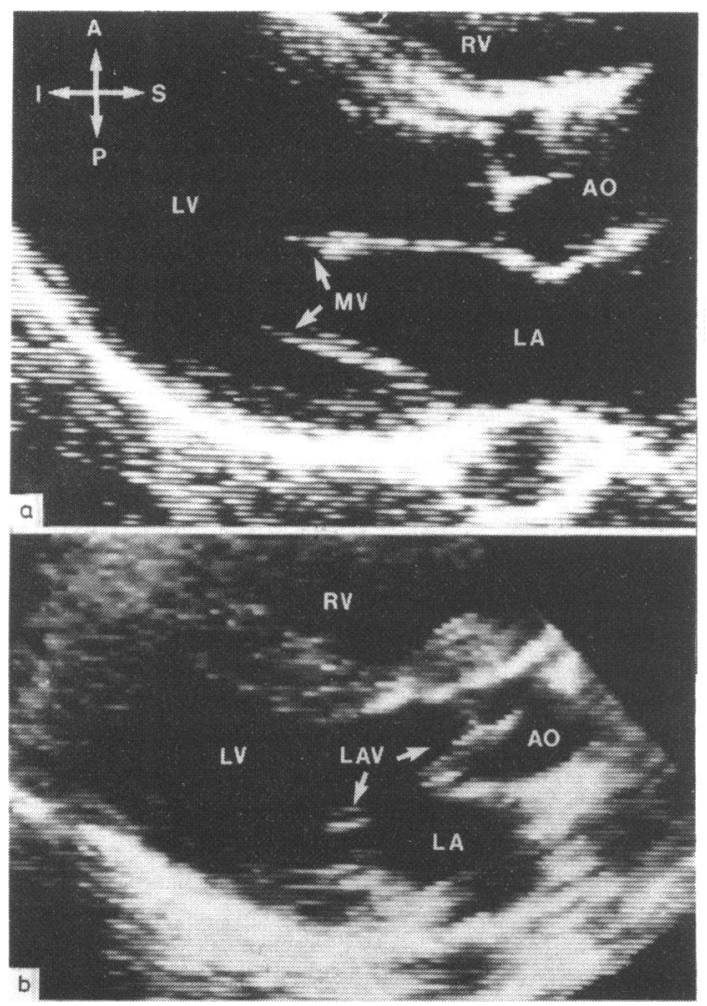

Fig. 4 (a) The upper picture is a parasternal long axis cut in a case with a normal atrioventricular junction and mitral valve. The mitral orifice points towards the apex of the left ventricle. (b) The lower picture is a parastermal long axis cut from a case with an atrioventricular septal defect. The lefi atrioventricular valve during diastole points towards the interventricular septum. LAV, left atrioventricular valve (for remaining abbreviations see Fig. 1 and 3). 
that the ventricular septal defect was identified in all cases. In the subcostal short axis cut the typical bileaflet pattern of the morphological mitral valve could be seen, with the tricuspid septal leaflet once again being firmly attached to the interventricular septum.

In the subcostal four chamber view, except in the case of very large ventricular septal defects, a cut could be obtained posterior to the ventricular septal defect in which offsetting of the atrioventricular valves caused by the muscular atrioventricular septum could be identified (Fig. 5a). When the transducer was then placed in the precordial four chamber cut the atrioventricular valves appeared at the same level, roofing a defect in the inlet septum (Fig. 5b).

\section{Atrioventricular discordance ( 31 cases)}

The apical four chamber cut disclosed a similar pattern to that found in patients with atrioventricular concordance, except that it was inverted (Fig. 6). A septal insertion of the tricuspid valve was seen in only 20 cases.

The precordial short axis cut was very difficult to obtain in all cases with atrioventricular discordance, because of the high frequency of a mid-line cardiac apex, which meant that the interventricular septum tended to lie behind the sternum.

In the parasternal long axis cut the morphologically left ventricle and mitral valve were seen to have the same appearance as in atrioventricular concordance. The left ventricular outflow tract, apart from evidence of obstruction in 20 cases, had normal mitral valve insertions.

In the subcostal four chamber cut the interventricular septum ran in a superoinferior direction in all cases, again reflecting the high frequency of a mid-line cardiac apex. The pulmonary and systemic veins were identified draining to their appropriate atrial chambers. The ventricular septal defect was visualised in all cases with this cut. The morphological tricuspid valve had septal insertions identified in all cases, while the mitral valve inserted towards the apex of the left ventricle, the interventricular septum being free from chordal attachments.

Only slight anterior angulation of the transducer from the four chamber cut was needed to demonstrate the pulmonary valve and artery, deeply wedged between the two atrioventricular valves. In this cut the ventricular septal defect was clearly visualised, as was the insertion of the tricuspid valve into the crest of the interventricular septum. Though the annulus of the mitral valve and the attachment of its leaflets towards the apex of the left ventricle was seen, in this cut the tricuspid annulus was not visualised. The insertion of the tricuspid valve into the crest of the ventricular septum lay inferior to the mitral annulus. Misin-

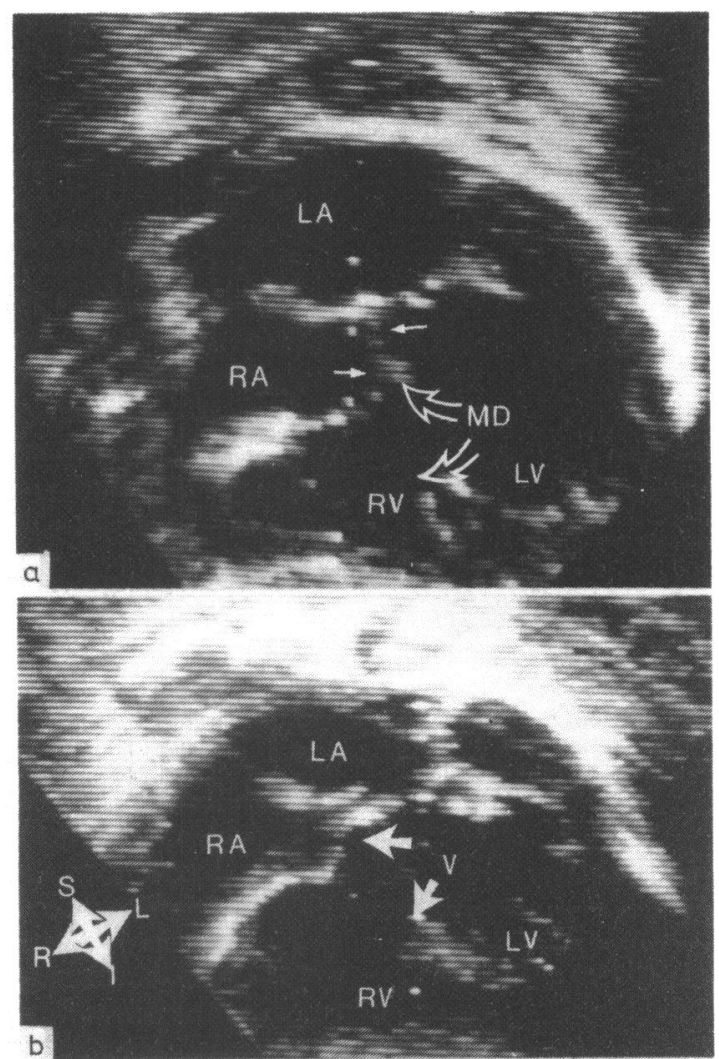

Fig. 5 (a) The upper picture is a subcostal four chamber cut in a case with a perimembranous inlet ventricular septal defect and associated muscular inlet ventricular septal defect. In this posterior cut, the atrioventricular muscular septum indicated by the small arrows, is intact. In addition the muscular inlet ventricular septal defect is visible. (b) The lower panel is a precordial four chamber cut from the same case. The atrioventricular valves are at the same level roofing the perimembranous inlet defect. MD, muscular defect (for remaining abbreviations see Fig. 1).

terpretation of this tricuspid insertion as tricuspid annulus would have led to an erroneous diagnosis of reversed offsetting, but the ventricular septal defect clearly lay superior to the tricuspid insertion, whereas it would lie inferior to the tricuspid annulus. In none of the cases studied was an atrioventricular muscular septum identified posterior to the ventricular septal defect.

In the long axis cut the left ventricular outflow tract appeared normal in all cases, apart from the presence of outflow tract obstruction in 23 cases.

In the short axis, the morphologically mitral valve was identified by its bileaflet pattern, whereas the tricuspid valve had septal insertions.

By combining the assessment of atrial situs from 


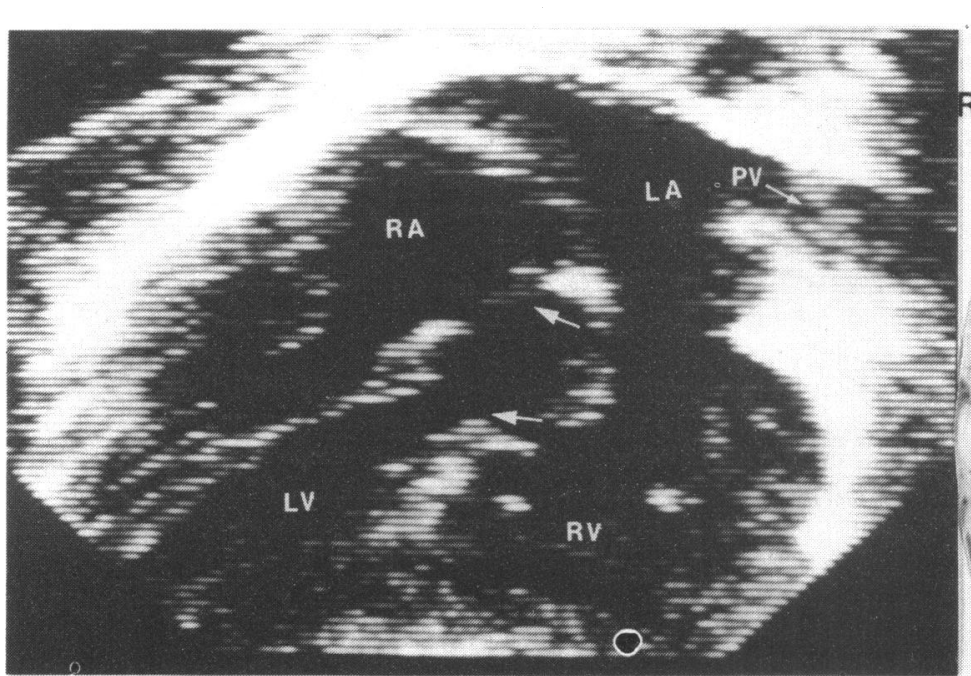

Fig. 6 The lefi picture is a precordial four chamber cut in a case with a perimembranous inlet ventricular septal defect and atrioventricular discordance. The mitral valve crosses the beam of the transducer, while the tricuspid orifice opens towards the centre of the cavity. The ventricular septal defect is indicated by the arrows. The specimen on the right is cut in the same plane indicating the echocardiographic features. PV, pulmonary vein (for remaining abbreviations see Fig. 1).
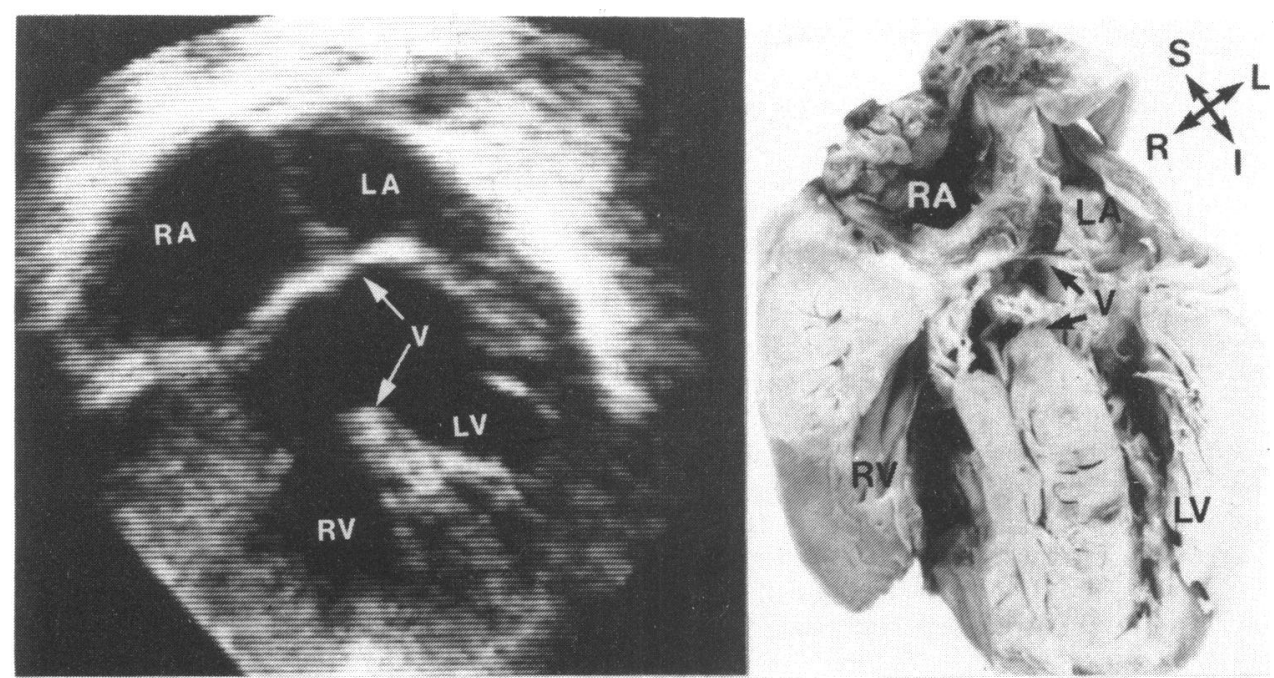

Fig. 7 The picture on the left is a precordial four chamber cut from a case with an atrioventricular septal defect and intact interatrial septum. The atrioventricular valves are at the same level and roof a defect in the inlet septum. The specimen on the right is from a case with an atrioventricular septal defect and intact interatrial septum. The defect in the inlet septum is indicated by the arrows. The anterior bridging leaflet is attached to the interatrial septum. (For abbreviations see Fig. 1.)

venous connections, filter films, and the relation of the abdominal aorta to the inferior vena cava, and the knowledge of the morphology of the connecting atrioventricular valves, the diagnosis of atrioventricular discordance could be made.
PERIMEMBRANOUS INLET DEFECT WITH ATRIOVENTRICULAR SEPTAL DEFECT ( 9 cases) In all cases the ostium primum septum was intact (Fig. 7). The atrioventricular valves were at the same्ष level, roofing a defect in the inlet septum. Both the 


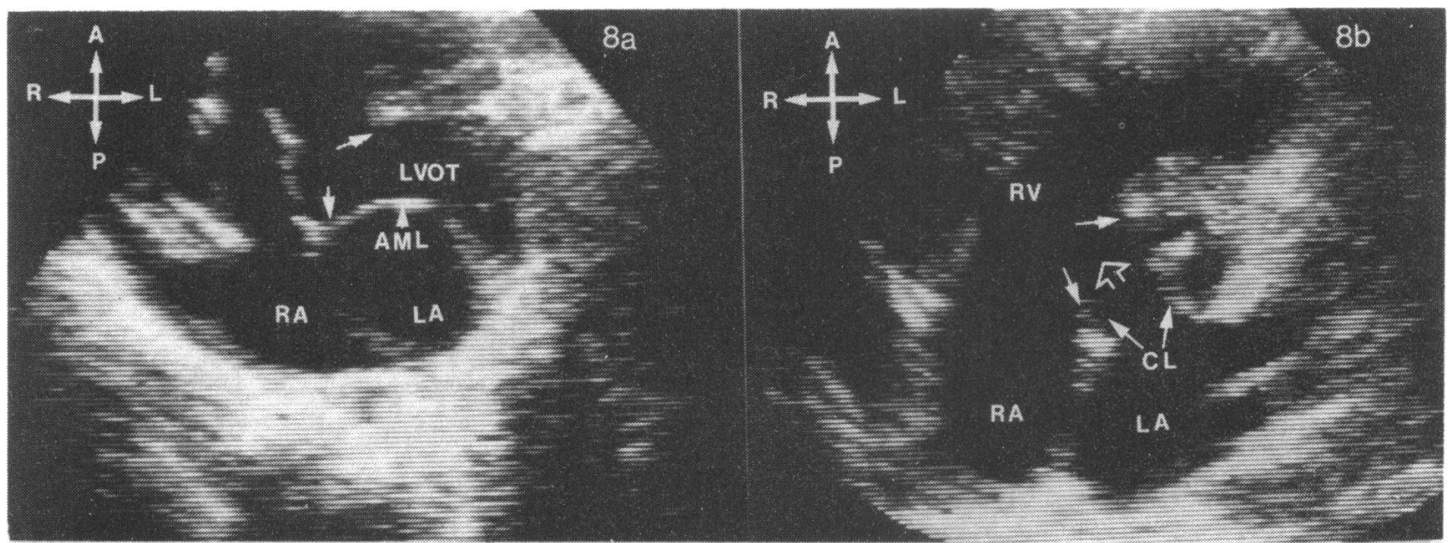

Fig. 8 (a) The picture on the left is a precordial short axis cut from a case with a perimembranous inlet ventricular septal defect and normal atrioventricular junction. The defect is indicated by the arrows. The normal anterior mitral leaflet can be seen. (b) The picture on the right is from a case with an atrioventricular septal defect, perimembranous inlet ventricular septal defect, and intact interatrial septum. The defect is indicated by the arrows. The "cleft" between the anterior and posterior bridging leaflets indicated by the open arrow points towards the right ventricle. $A M L$, anterior mitral leaflet; $C L$, cleft (for remaining abbreviations see Fig. 1 and 2).
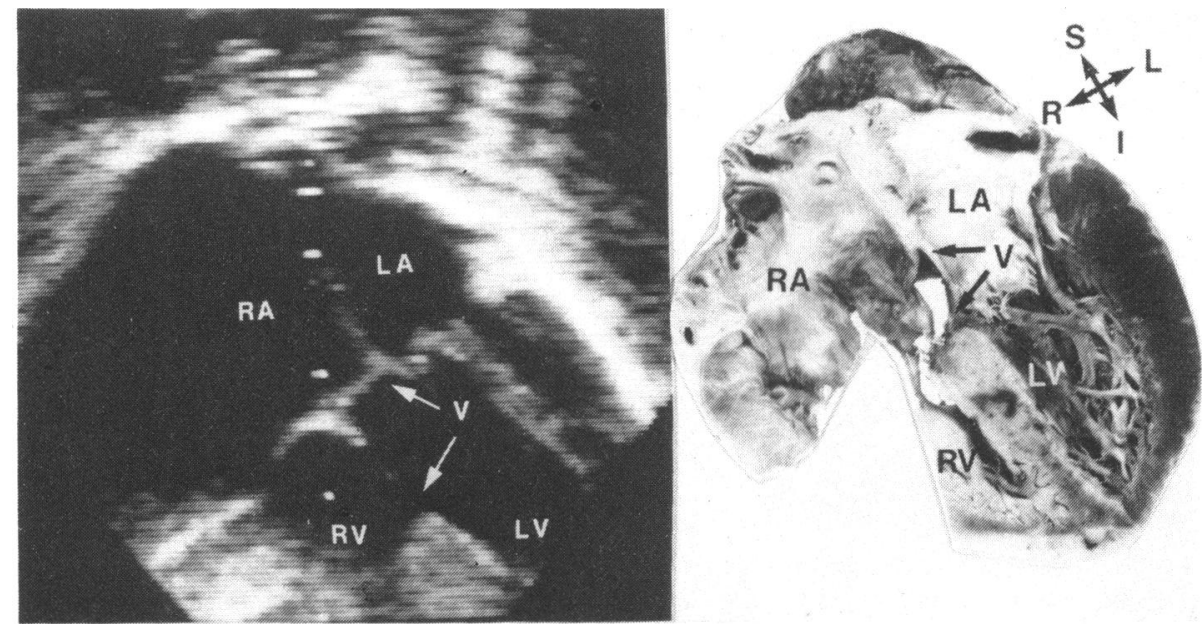

Fig. 9 The picture on the left is a precordial four chamber cut from a case with a perimembranous inlet ventricular septal defect and straddling tricuspid valve. There is some slight tricuspid annular overriding. The straddling valve is not seen in this anterior cut. The specimen on the right is from a case with a straddling tricuspid valve and perimembranous inlet ventricular septal defect. The straddling valve is also not seen in this anterior cut. In addition the mild degree of tricuspid annular overriding can be seen. (For abbreviations see Fig. 1.)

left and right sided valves appeared to open towards the centre of the cavity, whereas in hearts without an atrioventricular septal defect the mitral valve appears to be occluded by its anterior leaflet crossing the beam of the transducer (Fig. 6).

When the transducer was angled anteriorly from the four chamber cut, the posterior great vessel did not appear wedged between the atrioventricular valves (Fig. $2 b$ and $3 b$ ). It only appeared when the transducer was angled even more anteriorly.
In the precordial short axis cut the leaflets of the left sided valve appeared to separate as the scan head was angled more anteriorly, the division between them pointing towards the interventricular septum. This represented the "cleft" between the anterior and posterior bridging leaflets (Fig. 8 b).

In the parasternal long axis cut, the left ventricular outflow tract appeared to be narrow, with the left sided valve pointing towards the interventricular septum rather than the apex of the ventricle (Fig. 4b). 


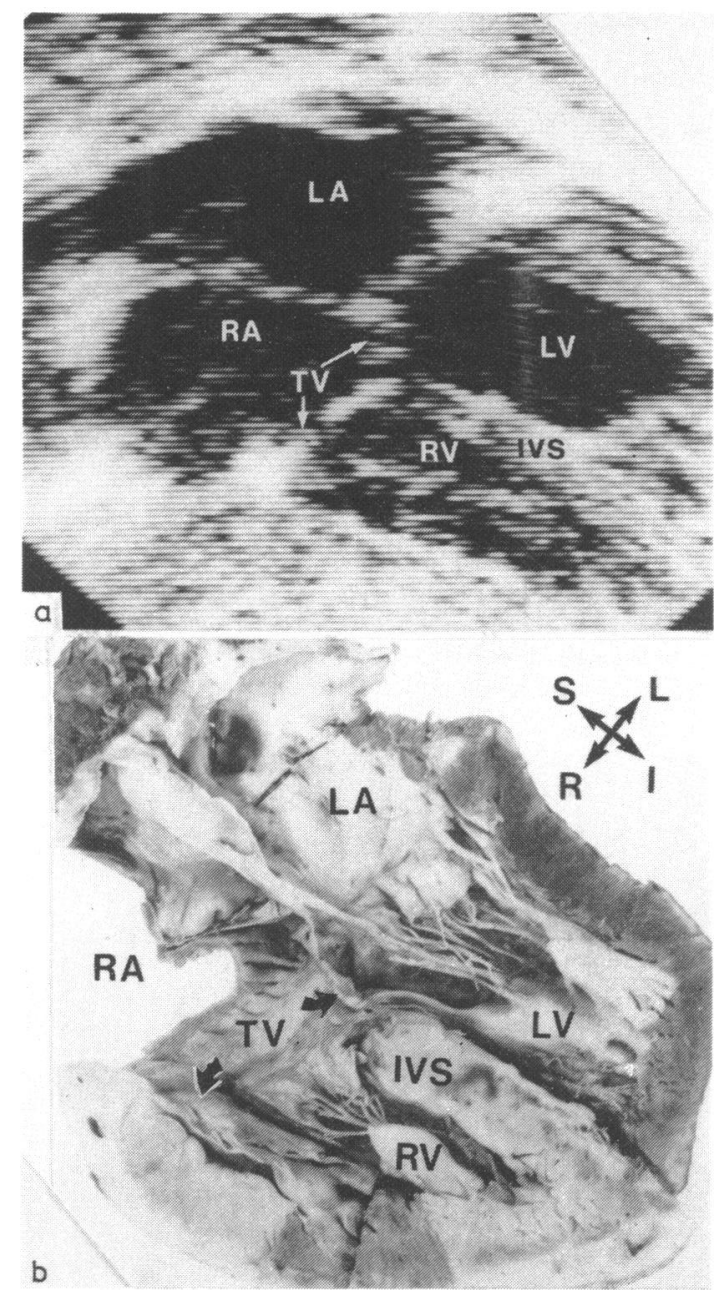

Fig. 10 The upper picture is a subcostal four chamber cut in a case with a perimembranous inlet ventricular septal defect and straddling tricuspid valve. The annular overriding is best seen in this cut. The lower picture is from a specimen with a straddling tricuspid valve and perimembranous inlet ventricular septal defect. The tensor apparatus from the tricuspid valve is in both ventricles. The maximum degree of annular overriding is seen in this cut. (For abbreviations see Fig. 1 and 3.)

A ventricular septal defect was visualised in all cases in the four chamber cut, with the atrioventricular valves appearing at the same level (Fig. 7).

In the subcostal long axis cut the anterior and posterior bridging leaflets appeared to have a foot in both ventricles, with the anterior bridging leaflet inserting into the left ventricular outflow tract and the posterior to the crest of the interventricular septum.

In the subcostal short axis the anterior and posterior bridging leaflets could again be seen in continuity across the crest of the interventricular septum,

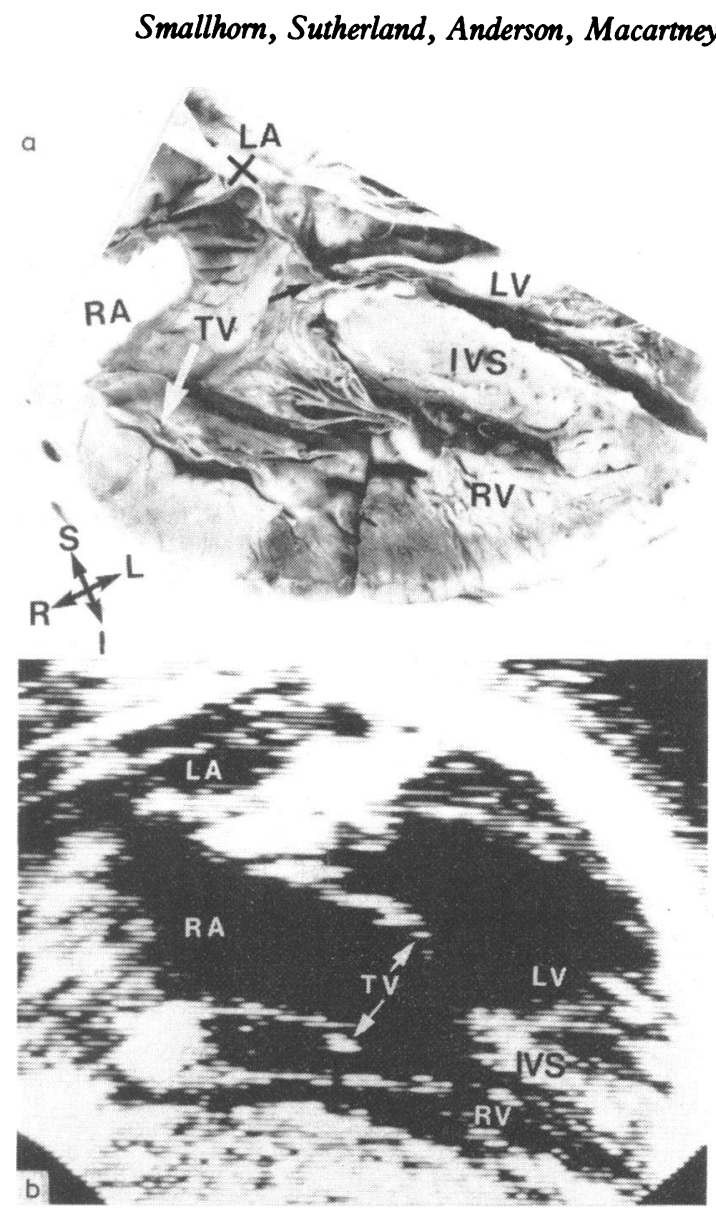

Fig. 11 The upper picture is an anatomical specimen cut posteriorly in a case with a straddling tricuspid valve and perimembranous inlet ventricular septal defect. The septum does not run to the crux of the heart. The lower picture is a subcostal cut, with the transducer angled to visualise only the tricuspid valve. The straddling tricuspid valve and the tricuspid annular overriding can be seen. The cross points to the crux of the heart. (For abbreviations see Fig. 1 and 3).

with the typical "cleft" between these structures. No 을 atrioventricular muscular septum could be identified $\frac{D}{0}$ posterior to the defect.

ONE OF TWO ATRIOVENTRICULAR VALVES STRADDLING PERIMEMBRANOUS DEFECT (10 cases)

In the precordial four chamber cut the atrioventricular valves were at the same level, roofing an inlet $\frac{0}{\Phi}$ defect (Fig. 9). Likewise, the ostium primum was $\stackrel{?}{+}$ intact in all cases. In eight cases a straddling right 0 (tricuspid) valve was visualised in this cut. The septal ${ }^{\circ}$ leaflet appeared to move through the ventricular sep- $\stackrel{\odot}{\circ}$ tal defect, the valve possessing tension apparatus in $\mathbb{D}$ both chambers (Fig. 10). The tension apparatus from 
the straddling valve was inserted towards the left ventricle in all cases. Two patients had a straddling left atrioventricular valve (mitral): again the leaflets moved through the ventricular septal defect during real-time. In all of those with a straddling right (tricuspid) atrioventricular and in one case with a straddling left (mitral) there was associated annular overriding.

With rightward and posterior angulation of the transducer in those patients with a straddling right atrioventricular valve, minor degrees of overriding could be detected by following the relation of the central fibrous body to the crest of the septum.

With anterior angulation of the transducer from the four chamber cut the posterior great vessel appeared wedged between the atrioventricular valves.

In the precordial short axis cut, the left sided valve had the same appearance as that seen in atrioventricular concordance without straddling (Fig. 8a).

In the parasternal long axis cut the left ventricular outflow tract appeared normal, as did the opening pattern of the morphological mitral valve. In six cases the straddling valve could also be seen in this cut.

In the subcostal four chamber cut the atrioventricular valves appeared at the same level in all cases. In those with a straddling tricuspid valve the posterior part of the interventricular septum appeared displaced anteriorly, such that the maximum amount of overriding could be readily visualised and the degree of straddling assessed (Fig. 11). In those with a straddling left atrioventricular valve (mitral), the interventricular septum ran to the crux of the heart, the straddling leaflets not being visualised in this cut, despite the defect involving the inlet septum.

In the subcostal long axis cut the mitral valve was inserted towards the apex of the left ventricle, the outflow tract having a normal appearance.

In the subcostal short axis cut the morphologically mitral valve had the typical bileaflet pattern. In addition, the additional tensor apparatus could be seen in the left ventricle in four cases with a straddling tricuspid valve.

\section{Discussion}

Before considering how these echocardiographic findings may be interpreted, it is essential to consider what is the precise nature of an "atrioventricular canal" type of isolated ventricular septal defect, since this is a matter about which there is much confusion. It is well recognised that a cardinal feature of true atrioventricular septal defects ("atrioventricular canal malformations" or "endocardial cushion defects") is that, provided no interventricular component of the defect is present, the atrioventricular valves are firmly attached to the ventricular septum at the same level, in contradistinction to the offsetting of the tricuspid and mitral valves so characteristic of the normal heart. ${ }^{6}$ This feature has been shown to be of great echocardiographic value. ${ }^{7}$ But in this circumstance, of course, the valves floor the septal defect. Van Mierop, ${ }^{8}$ however, described how a heart with all the characteristics of an atrioventricular septal defect could have a defect roofed by valves joined to the atrial septum at the same level. Such a heart would indeed be an isolated ventricular septal defect of "atrioventricular canal" type, and would possess the typical common atrioventricular junction rather than the normal mitral and tricuspid junctions. More frequently, perimembranous defects extending into the inlet ventricular septum ${ }^{9}$ with separate mitral and tricuspid annuli are termed "canal type" defects, and in these hearts too the valve leaflets would be attached to the central fibrous body at the same level. ${ }^{10}$ Another defect frequently considered as a canal defect is that associated with a straddling tricuspid valve. ${ }^{1112}$ Again, one feature of those anomalies is attachment of the valve leaflets to the atrial septum at the same level, but their distinguishing feature is the overriding valve annulus and the gross malalignment between atrial and ventricular septa. Finally, it is well recognised that ventricular septal defects in corrected transposition most frequently excavate the inlet septum $^{13}$ and are roofed by valves joined to the atrial septum at the same level. Despite this, inversion of the normal offsetting of these valves has been held as an echocardiographic characteristic of corrected transposition. ${ }^{2}$ All these varied defects have in common a deficiency of the inlet part of the ventricular septum and lack of the normal offsetting of the septal annular attachments of the atrioventricular valves, and may therefore be expected to give similar echocardiographic findings. With knowledge of the underlying anatomy, however, the echocardiographer should have at his fingertips the technique to distinguish the various types of inlet ventricular septal defect, and accurately to identify that elusive anomaly, the atrioventricular canal type of defect. As our study has shown, this is indeed the case. Though all the hearts studied had the septal leaflets of their atrioventricular valves attached to the atrial septum or central fibrous body at the same level, there were sufficient differences elsewhere to distinguish easily the different hearts.

The outstanding feature of the true atrioventricular septal defect with the potential only for intraventricular shunting and with two separate valve orifices was the fact that, though right and left orifices were separate, they were contained within a common atrioventricular junction. The left valve, as noted initially by Van Mierop ${ }^{8}$ and Carpentier ${ }^{14}$ was a trileaflet valve with different morphology from a normal mitral 
valve. This fact is readily demonstrated echocardiographically, where it can easily be shown that the anatomy is in no way replicated when a cleft is found in the anterior or aortic leaflet of an otherwise normally formed mitral valve. ${ }^{15}$ Thus, it is the unique anatomy of the entire atrioventricular junction which determines the diagnosis of an atrioventricular septal defect rather than the finding of the valve leaflets attached to the septum at the same level.

As shown, this latter finding is also present in isolated inlet perimembranous defects, in perimembranous inlet defects with atrioventricular discordance, and in inlet defects associated with straddling valves. But in none of these hearts is there the characteristic three leaflet left atrioventricular valve. Instead, the typical mitral valve morphology is readily identified (right sided in atrioventricular discordance) with an extensive mural leaflet and a much smaller anterior arterial leaflet. Often the septal defect can extend anteriorly into the trabecular or even the outlet parts of the septum and then mimic the true atrioventricular septal defect. But the junction is always different in the isolated defects, and often, when scanning towards the crux, a small area of atrioventricular muscular septum between offset valves can be detected posterior to the area of septal excavation. This is never found in the true atrioventricular septal defect. It may be thought that, because of the absence of offsetting in atrioventricular discordance, it would be difficult to distinguish the presence of the discordance atrioventricular connection. This was not the case, the nature of the distal insertions of the valve tension apparatus readily distinguishing the morphologically mitral from morphologically tricuspid valves. As our recent study devoted to echocardiographic features of atrioventricular discordance showed, ${ }^{16}$ the nature of the tension apparatus insertion was the single most helpful feature in the distinction of this atrioventricular connection. As we have observed, in the subcostal four chamber cut it is possible to confuse the tricuspid annulus with the tricuspid insertion. Though this will lead to a mistaken diagnosis of reversed offsetting, it will not affect the diagnosis of the ventricular morphology, since the very fact that the tension apparatus is inserted into the septum points to the fact that it originates from a tricuspid valve in a right ventricle. In straddling tricuspid valve the pathognomonic feature was the visualisation of tensor apparatus from one valve in both chambers, frequently but not always associated with malalignment between the atrial and the ventricular septum. This was seen even when the overriding right annulus was mostly connected to the morphologically right ventricular chamber, because in these hearts the ventricular septum never reaches to the crux. When the overriding annulus is connected mostly to the left ventricular chamber the echocar- diographic features are more reminiscent of double inlet to a left ventricular chamber; hardly surprising since that is the effective atrioventricular connection.

It can therefore be seen that identification of valvar offsetting is a useful, but a non-specific echocardiographic criterion. Of course, if valvar offsetting is positively identified superior to a defect of the inlet septum this is of major value, since the defect can almost certainly then be identified as a muscular inlet defect. Contrariwise, however, it must be remembered that valvar offsetting is not the rule even when there is atrioventricular concordance with an intact inlet septum. Our recent experience with necropsied hearts has shown that, with atrioventricular concordance and abnormal ventriculoarterial connections such as discordance or double outlet right ventricle, the valves tend to be attached to the septum at the same level without any intervening muscular atrioventricular septum. So, simply finding the valves attached.at the same level does not even mean that there is an inlet septal defect and may, unless particular care is taken to obtain multiple views of the same septal defect, lead to an erroneous diagnosis of its position in such cases. Nevertheless, recognition of the lack of offsetting is important precisely because it alerts the echocardiographer to the presence of one of the constellation of abnormalities that give rise to this appearance, and leads him to search for specific clues as to the precise abnormality present.

\section{References}

1 Foale RA, Somerville J. Ventricular morphology-twodimensional echocardiographic recognition in complex congenital heart disease (abstract). Br Heart f 1980; 43: $105-6$.

2 Hagler DJ, Tajik AJ, Seward JB, Edwards WD, Mair DD, Ritter DG. Atrioventricular and ventriculoarterial discordance (corrected transposition of the great arteries). Wide-angle two-dimensional echocardiographic assessment of ventricular morphology. Mayo Clin Proc 1981; 56: 591-600.

3 Soto B, Becker AE, Moulaert AJ, Lie JT, Anderson RH. Classification of ventricular septal defects. Br Heart $\mathcal{J}$ 1980; 43: 332-43.

4 Deanfield JE, Leanage R, Stroobant J, Chrispin AR, Taylor JFN, Macartney FJ. Use of high kilovoltage filtered beam radiographs for detection of bronchial situs $\omega$ in infants and young children. $B r$ Heart $\mathcal{F} 1980$; 44: 577-83.

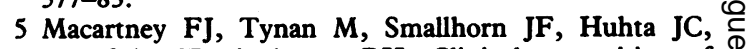
Deanfield JE, Anderson RH. Clinical recognition of atrial isomerism. In: Anderson RH, Shinebourne EA, Macartney FJ, Tynan M, eds. Paediatric cardiology 5. Edinburgh: Churchill Livingstone (in press).

6 Brandt PWT, Clarkson PM, Neutze JM, Barratt-Boyes BG. Left ventricular cineangiocardiography in endocar- 
dial cushion defect. (Persistent common atrioventricular canal.) Australas Radiol 1972; 16: 367-76.

7 Smallhorn JF, Tommasini G, Anderson RH, Macartney FJ. Assessment of atrioventricular septal defects by two dimensional echocardiography. Br Heart $\mathcal{f}$ 1982; 47: 109-21.

8 Van Mierop LHS. Pathology and pathogenesis of the common cardiac malformations. Cardiovasc Clin 1970; 2: 27-59.

9 Moulaert A. Anatomy of ventricular septal defect. In: Anderson RH, Shinebourne EA, eds. Paediatric cardiology 1977. Edinburgh: Churchill Livingstone, 1978: 113-24.

10 Sutherland GR, Godman MJ, Smallhorn JF, Guiterras $P$, Anderson RH, Hunter S. Ventricular septal defects. Two dimensional echocardiographic and morphological correlations. Br Heart $\mathcal{f}$ 1982; 47: 316-28.

11 Neufeld HN, Titus JL, DuShane JW, Burchell HB, Edwards JE. Isolated ventricular septal defect of the persistent common atrioventricular canal type. Circulation 1961; 23: 685-96.

12 LaCorte MA, Fellows KE, Williams RG. Overriding tricuspid valve: echocardiographic and angiocardiographic features. Am f Cardiol 1976; 37: 911-9.
13 Becker AE, Anderson RH. Conditions with discordant atrioventricular connections-anatomy and conducting tissues. In: Anderson RH, Shinebourne EA, eds. Paediatric cardiology 1977. Edinburgh: Churchill Livingstone, 1978: 184-97.

14 Carpentier A. Surgical anatomy and management of the mitral component of atrioventricular canal defects. In: Anderson RH, Shinebourne EA, eds. Paediatric cardiology 1977. Edinburgh: Churchill Livingstone, 1978: 477-90.

15 Smallhorn JF, de Leval M, Stark J, et al. Isolated anterior mitral cleft. Two dimensional echocardiographic assessment and differentiation from "clefts" associated with atrioventricular septal defect. Br Heart $\mathcal{f}$ 1982; 48:109-16.

16 Sutherland GR, Smallhorn J, Rigby ML, Anderson RH, Hunter S. The 2-D echocardiographic spectrum of atrioventricular discordance (abstract). Circulation 1981; 64, suppl IV: IV-166.

Requests for reprints to Professor FJ Macartney, The Hospital for Sick Children, Great Ormond Street, London WCIN 3JH. 